\title{
Raman-scattering depth profile of the structure of ion-implanted GaAs
}

\author{
M. Holtz, ${ }^{*}$ R. Zallen, and O. Brafman ${ }^{\dagger}$ \\ Department of Physics, Virginia Tech, Blacksburg, Virginia 24061 \\ S. Matteson ${ }^{\ddagger}$ \\ Central Research Laboratories, Texas Instruments Inc., Dallas, Texas 75265
}

(Received 13 May 1987)

\begin{abstract}
We have carried out an extensive Raman-scattering investigation of the structure of berylliumimplanted gallium arsenide. Single-crystal $\mathrm{GaAs}$ was bombarded with $45-\mathrm{keV} \mathrm{Be}^{+}$ions, and backscattering Raman measurements were made, prior to any anneal, as a function of ion fluence, laser photon energy, and depth (via chemical-etch removal of surface layers). Line-shape and intensity analyses of the observed first-order Raman spectra, especially of the longitudinal-optical- (LO) phonon line (which is superimposed on the broad spectral signature of amorphous GaAs), support a structural model of the implantation-induced damage layer as a fine-scale mixture of amorphous and crystalline GaAs. The etch studies yield a structural depth profile in terms of the depth dependence of the amorphous volume fraction (derived from measured scattering intensities) and of the characteristic crystallite size. The first $1500 \AA$ is a high-damage layer having nearly constant structure; this is followed by a structurally graded transition region in which the crystalline volume fraction and the crystallite size smoothly increase until the bulk crystal is reached at about $4000 \AA$. For a fluence of $5 \times 10^{14}$ ions $/ \mathrm{cm}^{2}$, the near-surface high-damage plateau is characterized by an amorphous volume fraction of 0.25 and a crystallite size of $60 \AA$. This plateau begins at the surface; there is no evidence of the near-surface decrease in disorder which appears in some commonly used theoretical simulations. Varying the laser photon energy from 1.55 to $2.71 \mathrm{eV}$ reveals that the $\mathrm{LO}$ intensity (arising from the crystalline component) increases at both ends of this spectral range. The intensity increase at low photon energies reflects the increasing optical penetration depth (i.e., effective scattering volume), but the increase at high photon energies signifies a real rise in the scattering efficiency. We interpret this as a resonance-Raman effect associated with the approach toward the $E_{1}$ interband transition. This resonance is partially quenched as the crystallite size is decreased for heavily implanted samples.
\end{abstract}

\section{INTRODUCTION}

When energetic ions are implanted into a semiconductor for doping purposes, damage occurs to the host lattice because of inelastic collisions between the incident ions and the host atoms. The spatial rate of energy loss is a function of the energy, resulting in a depth dependence to the damage. High-energy ions have a reduced nuclear scattering cross section and are slowed down primarily through electronic scattering. As the ions lose energy, collisions with the substrate ions become more frequent, resulting in structural disorder. The atomic scale structure of the near-surface damage layer is not well understood. Depending upon the implant conditions (ion energy and mass, ion fluence, target, target temperature, etc.), it may consist of amorphous regions and/or microcrystals and/or disordered crystalline regions.

Gallium arsenide is a high-mobility, direct-band-gap semiconductor which is useful for a variety of applications. Doping GaAs via ion bombardment is widely used, and extensive theoretical treatments have been developed to estimate the depth distribution of the dopant ions and the lattice damage. ${ }^{1,2}$ Beryllium is an implant species often used to produce $p$-type GaAs. ${ }^{3} \mathrm{Be}$ fore the doping process is complete, the impurity ions must substitute onto lattice sites and the damage caused by implanting must be annealed out. The structure of the damage layer in beryllium-implanted GaAs, prior to annealing, is the focus of the present study.

Raman scattering is a nondestructive technique which is sensitive to both structural and electronic characteristics. In opaque semiconductors (laser photon energy above the band gap) the scattering comes from a thin surface layer, so that the technique provides an excellent probe of near-surface structure. Raman spectra have recently been used to characterize ion-implanted GaAs. ${ }^{4-7}$ In particular, Tiong et $a l .{ }^{4}$ have used a "spatial correlation" model similar to that of Richter et $a l^{8}$ to estimate crystallite sizes for arsenic implants, based on the measured peak position and linewidth of the longitudinaloptical- (LO) phonon line in the Raman spectrum. Other optical techniques have been used to study the depth dependence of ion damage, ${ }^{9-11}$ but only Raman scattering discriminates between the separate crystalline and amorphous spectral components. The combination of Raman scattering with chemical-etch removal of nearsurface layers has not been previously exploited, and this approach is emphasized in the present investigation. The effectiveness of this approach is demonstrated by our results, especially with respect to the determination of 
quantitative measures of the structural depth profile of implantation-induced damage.

In this paper we report the results of an extensive Raman-scattering investigation of the structure of the damage layer that is produced in GaAs by bombardment with $\mathrm{Be}^{+}$ions. Depth profiles of the structural character of the implanted layer were obtained by analyzing the spectral changes which occur as a function of both chemical-etch time and laser photon energy. The observed spectral changes are interpreted in terms of a depth-dependent crystalline-amorphous mix and crystallite size distribution; these new, Raman-derived profiles constitute the structural information which is our main focus. Information is also obtained about the optical absorption in partially amorphized GaAs. In addition, we have observed damage-dependent resonance-Raman effects; these new effects are attributed to the influence of crystallite size.

\section{EXPERIMENT}

The starting material was chromium-doped, Czochralski-grown, semi-insulating, (100)-oriented, single-crystal GaAs. Prior to implanting, the polished wafers were etched in 8:1:1 $\mathrm{H}_{2} \mathrm{SO}_{4}: 30 \% \mathrm{H}_{2} \mathrm{O}_{2}: \mathrm{H}_{2} \mathrm{O}$ to yield samples free of residual damage and of excellent optical quality. $45-\mathrm{keV}^{9} \mathrm{Be}^{+}$ions were implanted at room temperature, incident $9^{\circ}$ from $(100)$ to reduce channeling. Fluences ranged from $10^{13}$ to $5 \times 10^{14} \mathrm{~cm}^{-2}$.

The depth profiling was accomplished using a weak acidic solution of $1: 1: 100 \mathrm{H}_{2} \mathrm{SO}_{4}: 30 \% \mathrm{H}_{2} \mathrm{O}_{2}: \mathrm{H}_{2} \mathrm{O}^{12}$ Etching was done at room temperature for up to $6 \mathrm{~min}$, in 1min steps. The etch was calibrated by masking a narrow strip with polymethyl methacrylate photoresist prior to successive etches. The resist was then removed and the step height of the masked area was determined using a stylus profilometer (Tencor Instruments, Alpha-Step) and by Tolansky interferometry. ${ }^{13}$ Because the damage is expected to affect the etch rate, both pristine crystal and heavily implanted $\left(5 \times 10^{14} \mathrm{~cm}^{-2}\right)$ samples were measured. Depth versus etch time results for these samples are shown in Fig. 1. The unimplanted crystal is seen to etch uniformly at a rate of about $530 \AA / \mathrm{min}$, in excellent agreement with a reported result. ${ }^{14}$ The ion-damaged material etches more quickly, approximately $660 \AA / \mathrm{min}$, and is expected to etch at the same rate as the crystal beyond the damaged region.

Raman spectra were collected at room temperature, using a SPEX 1403 double monochromator and a cooled GaAs photocathode photomultiplier as a detector in the photon counting mode. Excitation was produced using either a krypton-ion or an argon-ion laser. This enabled us to vary the source photon energy (wavelength) from $1.55 \mathrm{eV}(7993 \AA)$ to $2.71 \mathrm{eV}(4579 \AA)$, thereby allowing us to change the optical penetration depth into the sample by as much as an order of magnitude in crystalline GaAs. ${ }^{15}$ Laser power was kept below $20 \mathrm{~mW}$ at the sample.

A near-backscattering geometry was used with the excitation incident at about $40^{\circ}$ from the normal outside the sample, corresponding to a maximum internal angle of

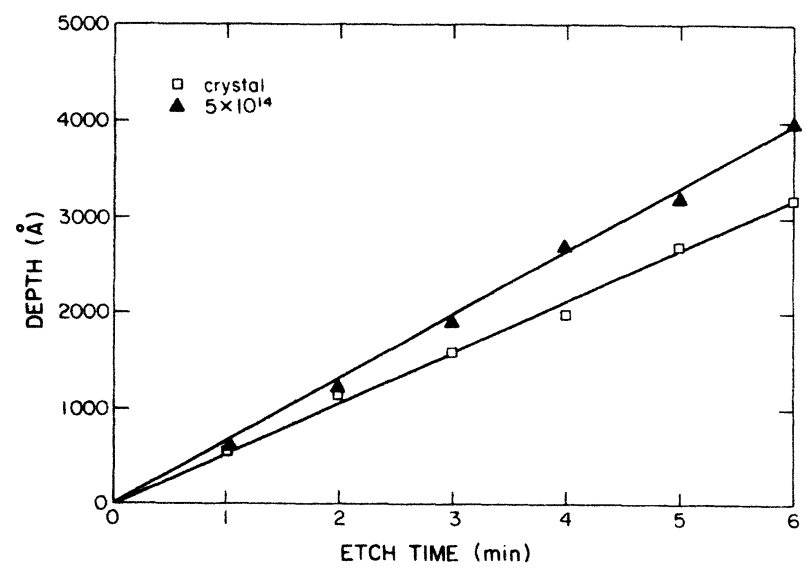

FIG. 1. Calibrations of the weak acidic etch used to depth profile for the crystalline starting material and the most heavily implanted sample studied $\left(5 \times 10^{14} \mathrm{~cm}^{-2}\right)$.

$10^{\circ}$. Laser polarization was in the scattering plane and scattered light was not analyzed. For this arrangement, along a (100) direction, scattering by LO phonons is allowed, while the transverse optical (TO) phonon is forbidden. ${ }^{16}$ In order to compare scattering intensities between different samples and for different photon energies, an external, transparent, reference standard was used. $\mathrm{CaF}_{2}$ is transparent throughout the visible, and has constant scattering efficiency below $5 \mathrm{eV} .^{17}$ We used the $\mathrm{CaF}_{2}$ $322-\mathrm{cm}^{-1}$ line as the reference standard. Because of the proximity of the $322-\mathrm{cm}^{-1}$ line to the GaAs LO line (291 $\mathrm{cm}^{-1}$ ), and because the $\mathrm{CaF}_{2}$ signal is very strong, spectra could not be collected with the sample and the reference simultaneously in place. Instead, a kinematicmount replacement procedure was developed, and consistent, repeatable values were obtained in this manner. The use of an intensity reference allows us to compare intensities for various etch times, and for particular samples across a wide range of photon energies. The precision is not expected to be better than $10 \%$.

\section{DAMAGE-LAYER DEPTH PROFILE}

Figure 2 shows full first-order Raman spectra of (100) $\mathrm{GaAs}$ before implanting and following various implants of $45-\mathrm{keV} \mathrm{Be}^{+}$ions. Here Stokes Raman-scattering intensity is plotted versus frequency shift $-\Delta \bar{v}$ in wave number units $\left(1 / \lambda\right.$ in $\left.\mathrm{cm}^{-1}\right)$ from the laser frequency. The spectra of Fig. 2 (as well as those of Figs. 3 and 4) were obtained using the argon green line: $\lambda_{L}=5145 \AA$, $\hbar \omega_{L}=2.41 \mathrm{eV}$. The intensity scale for all of the curves presented on a given figure have the same normalization with respect to the $\mathrm{CaF}_{2}$ standard.

The crystal spectrum at the top of Fig. 2 exhibits a strong LO line at $291 \mathrm{~cm}^{-1}$, accompanied by a weak TO line at $268 \mathrm{~cm}^{-1}$. Although the TO is forbidden in backscattering along (100), we observe it here because we are not in a strict backscattering geometry and because the scattered light is collected in a cone about (100). With increasing fluence, the LO line is seen to decrease in intensity, downshift in energy, and broaden in linewidth. This 


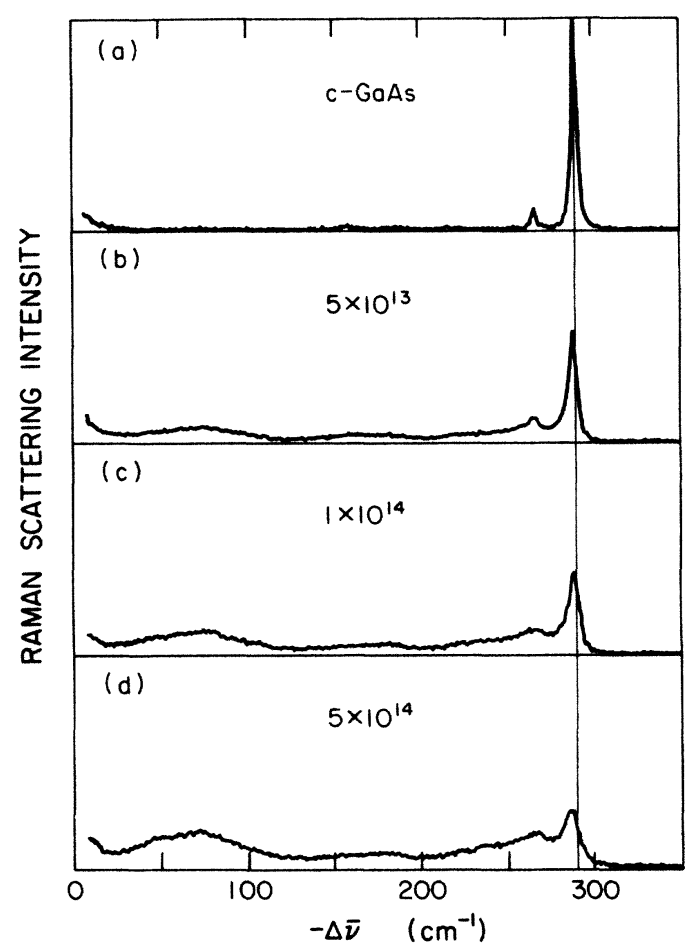

FIG. 2. Full Raman spectra (spanning the first-order lattice fundamental regime from 0 to $300 \mathrm{~cm}^{-1}$ ) for crystalline $\mathrm{GaAs}$ and for $\mathrm{GaAs}$ implanted with increasing fluences of $45-\mathrm{keV}$ $\mathrm{Be}^{+}$. Each curve is labeled in units of ions $/ \mathrm{cm}^{2}$. These spectra were taken with 5145 - $\AA$ excitation; intensities are normalized to the $\mathrm{CaF}_{2}$ standard.

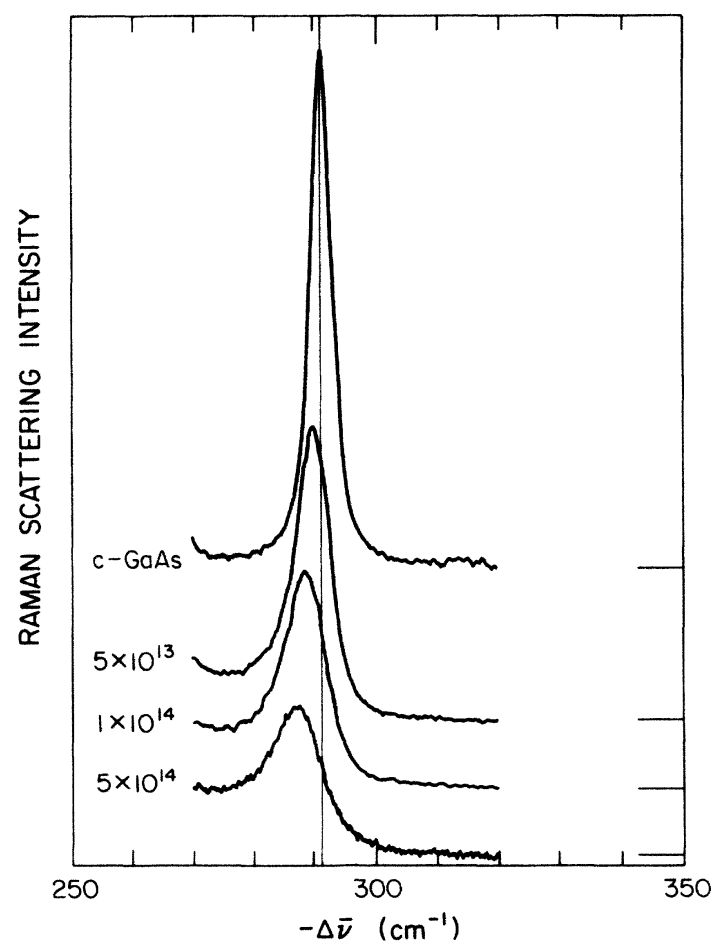

FIG. 3. Detailed spectra in the vicinity of the crystal LO line, for the same samples as in Fig. 1. All curves are plotted on the same vertical scale $\left(\mathrm{CaF}_{2}\right.$ standard), with the zero intensity (taken at $320 \mathrm{~cm}^{-1}$ ) displaced for clarity.

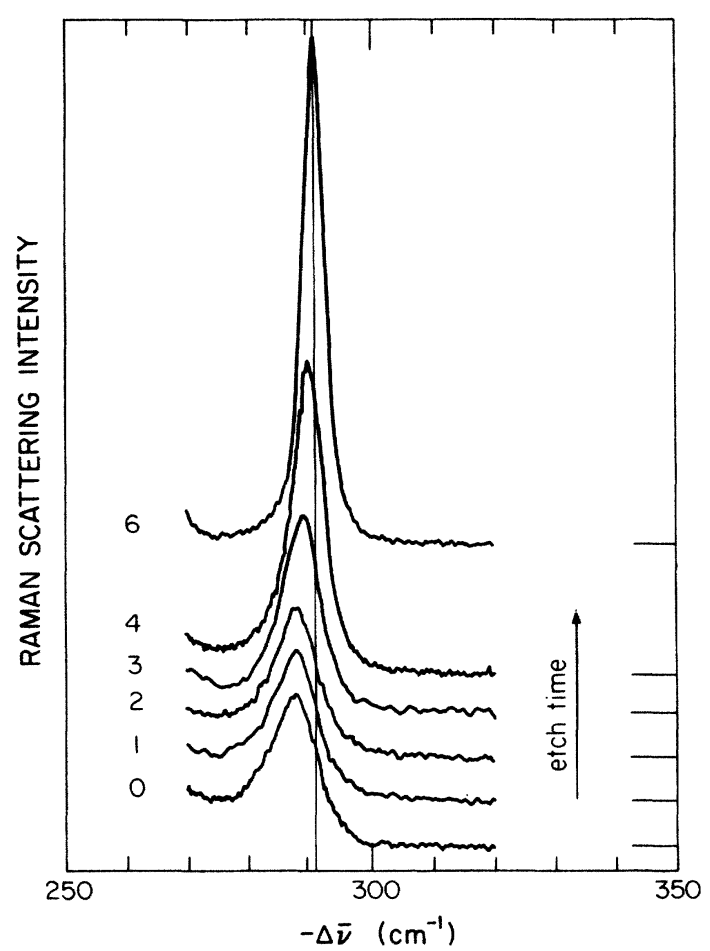

FIG. 4. LO spectra (5145- $\AA$ source) of the $5 \times 10^{14}-\mathrm{cm}^{-2} \mathrm{im}$ plant for various etch times shown on graph in min. By 5 to 6 min of etch time the spectrum is indistinguishable from that of the crystal. All are on the same vertical scale $\left(\mathrm{CaF}_{2}\right.$ standard).

is due to the implantation-induced change in the crystalline portion from the bulk crystal to a collection of crystallites. A decrease in crystallite size limits the correlation length $L$ of the optical phonon and thereby replaces the strict $\mathbf{k}=\mathbf{0}$ crystal selection rule with a more permissive range $(\Delta k \sim 1 / L)$ of LO phonon $\mathbf{k}$ values. ${ }^{8}$

Also evident in Fig. 2 are additional low-energy bands which increase in intensity with higher fluence. These broad bands comprise the Raman signature of amorphous GaAs. ${ }^{4,5,18,19}$ Implanting, then, produces a nearsurface damage region which consists of an amorphousmicrocrystalline mixture. The simultaneous appearance of spectral signatures of both the crystalline form ( $\mathrm{LO}$ line, albeit shifted and broadened) and the amorphous form (three-band continuum from 0 to $300 \mathrm{~cm}^{-1}$ ) supports this picture.

Much of the analysis to follow in this article relies upon our ability to extract accurate information about the detailed evolution of the narrow LO line. This capability is demonstrated in Fig. 3, which displays an expanded view of the influence of ion fluence on the LO line. This figure shows that our experiments permit us to clearly observe and quantitatively determine the bombardment-induced changes in the spectral characteristics (position, linewidth, intensity) of this band.

In order to obtain accurate values for the spectral parameters of the LO line from measurements such as those shown in Fig. 3, it is necessary to correct for the contribution of the broad bands of amorphous GaAs. This is especially important at higher doses, for which the amorphous-GaAs spectral component is substantial. 
(Note the low-frequency wing on the bottom curve of Fig. 3.) To do this, the full spectrum $\left(20-300 \mathrm{~cm}^{-1}\right.$ as in Fig. 2) was used to estimate the strength of the amorphous component, and then the thus-normalized spectrum of amorphous GaAs (Ref. 20) was subtracted from the data in the LO region.

The variation with etch time of the LO spectrum (raw data) is shown in Fig. 4 for the $5 \times 10^{14}-\mathrm{cm}^{-2}$ implant. The spectra for 0,1 , and $2 \mathrm{~min}$ of etch (corresponding to etch depths of 0,660 , and $1320 \AA$ ) are identical in all respects (in fact, the full spectra are identical), indicating that the level of damage is constant in this region. After $2000 \AA$ have been etched away, the LO line begins to narrow, while shifting toward higher frequency and growing in intensity, indicating less damage. By 3500-4000 $\AA$ the spectrum is that of the pristine crystal, indicating that the full damage layer has been etched away to expose the undamaged crystalline substrate.

The similarity of the curves for etch times of 0,1 , and 2 min shows that the optical penetration depth $d_{\text {opt }}$ is smaller than the depth corresponding to $1 \mathrm{~min}$ of etch $\left(d_{\text {opt }}<660 \AA\right)$. In the present context, in which the scattered light must return along the same sample length as that penetrated by the laser light, $d_{\text {opt }}$ is taken to be $1 /(2 \alpha)$, where $\alpha$ is the optical absorption coefficient. At $2.41 \mathrm{eV}, d_{\text {opt }}$ is $550 \AA$ in crystalline GaAs (Ref. 15) and about $140 \AA$ in amorphous $\mathrm{GaAs},{ }^{9}$ values consistent with our finding that $d_{\text {opt }}<660 \AA$ in the mixed phase.

Etch profiles like those in Fig. 4 for $5 \times 10^{14} \mathrm{~cm}^{-2}$ were obtained for $5 \times 10^{13}$ - and $1 \times 10^{14}-\mathrm{cm}^{-2}$ implants under

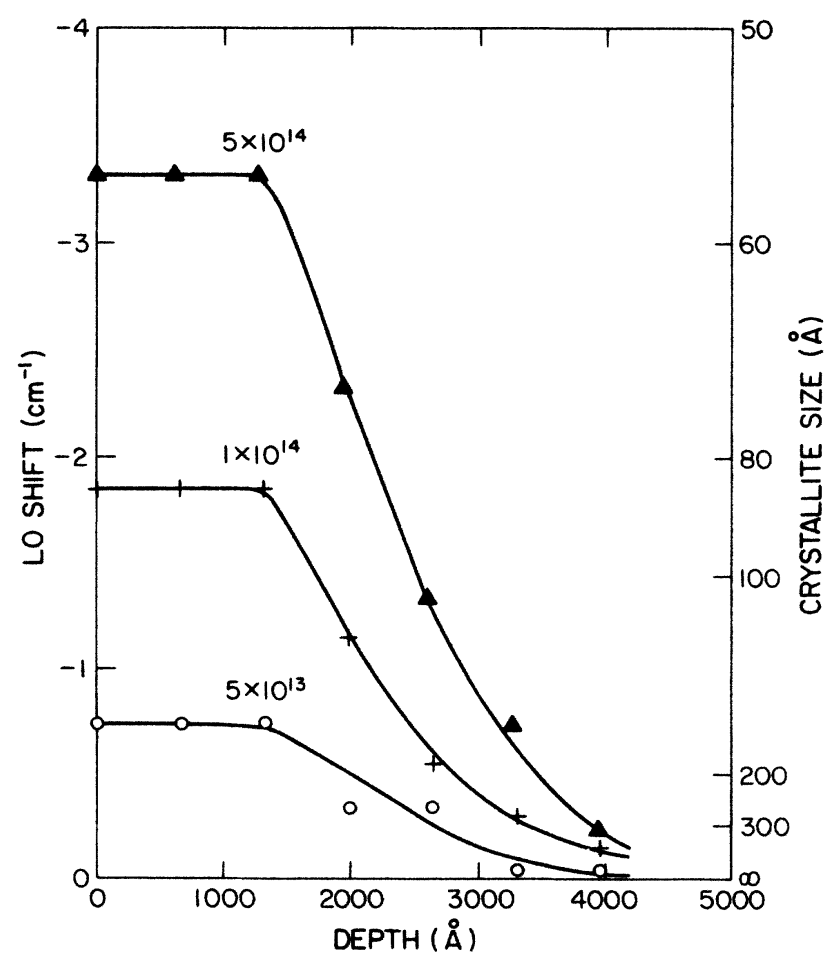

FIG. 5. LO downshift from the crystalline LO frequency vs etch depth for three fluences: $5 \times 10^{13}, 1 \times 10^{14}$, and $5 \times 10^{14}$ $\mathrm{cm}^{-2}$. The right-hand vertical scale is the crystallite size $L$, associated with the frequency shift on the left, taken from Ref. 4. identical conditions. All of these results are summarized in Figs. 5, 6, and 7. Figure 5 displays the shift in the peak position of the LO line $\left[\Delta v_{\mathrm{LO}}\right.$ (damaged) $-\Delta v_{\mathrm{LO}}$ (crystal)] as a function of etch depth. Figure 6 shows the LO linewidth (corrected for instrument resolution) versus depth, with the crystalline linewidth at 3.2 $\mathrm{cm}^{-1}$. Relating these results to average microcrystallite size (right-hand border of Fig. 5) using the analysis of Tiong et al., ${ }^{4}$ we find consistency between Figs. 5 and 6 and their results. Figures 5 and 6 can thus be used to estimate a characteristic crystallite size as a function of depth, and this will be done below. Several main features are already clear at this point. Our experiments reveal a total damage-layer depth (for $45-\mathrm{keV} \mathrm{Be}^{+}$implants) of about $4000 \AA$, and a high-damage plateau region of constant high damage extending from the surface to a depth of about $1500 \AA$. The amplitude of the damage profile naturally increases with increasing fluence, but the depth-dependent shape is insensitive to fluence.

Figure 7 presents the LO integrated intensity observed as a function of etch depth for each of the same three fluences. These measurements of absolute Ramanscattering intensities are difficult; they rely upon repeated sample substitutions and comparisons to the $\mathrm{CaF}_{2}$ standard. The appreciable scatter is therefore not surprising. However, the pattern is quite clear and is consistent with Figs. 5 and 6. Specifically, the total depth of damage is the same for all three fluences (of course, the degree of damage increases with fluence), and all three implants exhibit a similar near-surface high-damage plateau region. Within the high-damage plateau, the LO intensity is

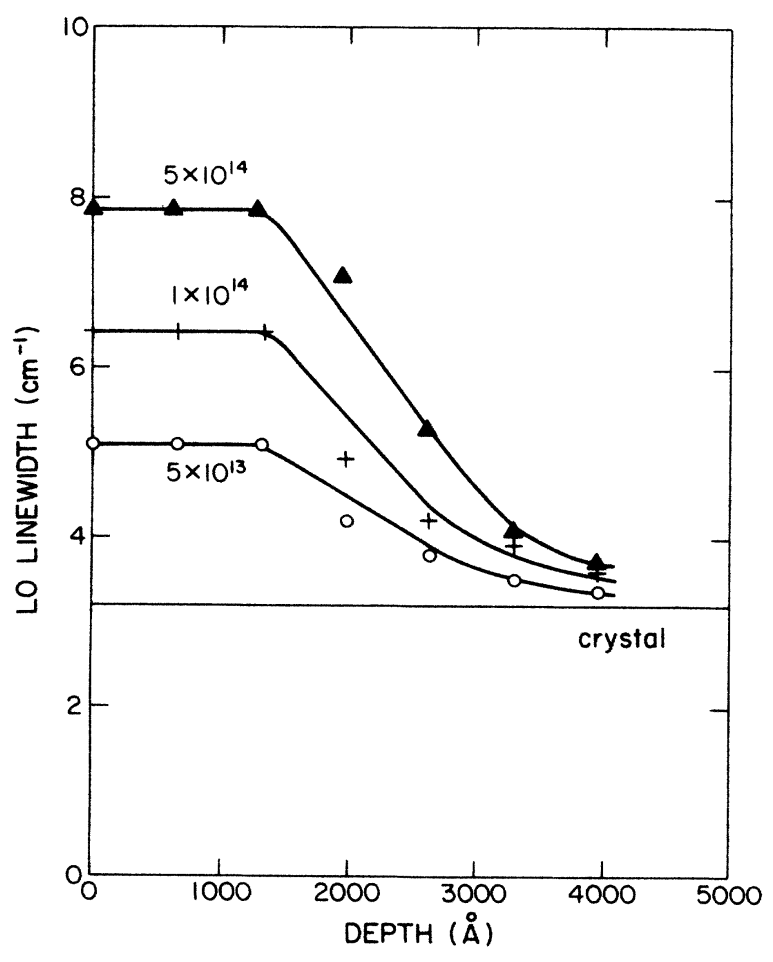

FIG. 6. LO linewidth vs etch depth for the same three implant fluences as in Fig. 5. The crystalline linewidth of $3.2 \mathrm{~cm}^{-1}$ is indicated by the horizontal line. 


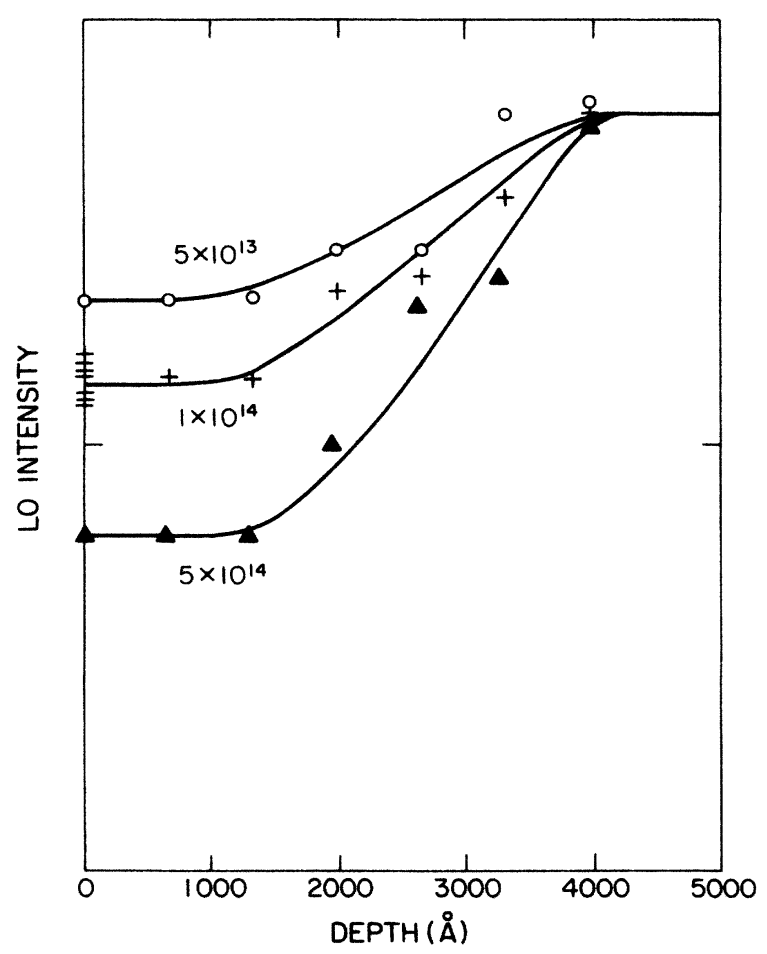

FIG. 7. LO intensity (linewidth times peak height), relative to $\mathrm{CaF}_{2}$, vs etch for the same sequence of fluences and etches as in Figs. 5 and 6. The source used was 5145- $\AA$ laser light. The curves were generated using Eqs. (1), (3), and (4), as discussed in the text. Precision can be seen from the repeated data points plotted for $1 \times 10^{14} \mathrm{~cm}^{-2}$, no etch.

small because the fraction of the scattering volume which is still crystalline is small and because the penetration depth is also reduced.

The curves in Figs. 5 and 6 are simply smooth representations of the data points, unlike the curves in Fig. 7, which are the results of theoretical calculations based on a simple model of the damage layer. Since we expect the optical properties to vary with the crystalline-amorphous mix, which in turn varies with depth, the measured intensity of the crystalline component of the spectrum (i.e., the LO integrated intensity) is related to the volume probed by

$$
I_{c} \sim \int_{0}^{\infty} d x f_{c}(x) \exp \left[-2 \int_{0}^{x} \alpha\left(x^{\prime}\right) d x^{\prime}\right],
$$

where $x\left(x^{\prime}\right)$ is the depth from the surface, $f_{c}(x)$ is the crystalline volume fraction at depth $x$, and $\alpha(x)$ is the optical absorption coefficient at $x$. The factor of 2 in the exponent arises from attenuation of the laser light traveling a distance $x$ into the material, and attenuation of the scattered light as it travels back out distance $x$. Differences between the crystalline and damaged GaAs reflectances were found to be small, a point which we will return to. We assume the same $\alpha$ for the scattered light as the laser beam (the two photon energies differ by only $1 \%$ ), and we assume that losses due to Raman scattering are small compared to optical absorption. The sample thickness is taken as infinite compared to $d_{\text {opt }}$.
Equation (1) is a generalization of the homogeneous, opaque case, for which $I \sim d_{\text {opt }} \sim 1 /(2 \alpha)$, to inhomogeneous systems of varying microstructure and optical absorption. Our view of the implanted layer as a mixed crystalline-amorphous system requires that

$$
\alpha=f_{c} \alpha_{c}+f_{a} \alpha_{a},
$$

where $\alpha_{c}$ and $\alpha_{a}$ are, respectively, the absorption coefficients of the crystalline and amorphous components. The effective medium approximation (EMA) was also used to estimate $\alpha$, using the EMA form of Aspnes ${ }^{21}$ for the dielectric function of a two-phase medium. We found that the results were the same, to within $1 \%$, as the results obtained using Eq. (2). Thus this simple equation is quite adequate for our volume fraction analysis. Here, $f_{a}$ is the amorphous volume fraction.

Using $f_{c}+f_{a}=1$, Eq. (2) becomes

$$
\alpha(x)=f_{c}(x) \alpha_{c}+\left[1-f_{c}(x)\right] \alpha_{a}
$$

and Eq. (1) depends only on $f_{c}(x)$ since $\alpha_{c}$ and $\alpha_{a}$ are known. The curves in Fig. 7 are generated from Eq. (1) using

$f_{c}= \begin{cases}f_{c}(0), & 0-1600 \AA \\ f_{c}(0)+\left[1-f_{c}(0)\right] \frac{x-1600(\AA)}{2600(\AA)}, & 1600-4200 \AA \\ 1, & \geq 4200 \AA\end{cases}$

where the portion linear in $x$ is a continuous interpolation from the end of the high-damage plateau [throughout which $f_{c}=f_{c}(0)$, the near-surface value] to the start of the undamaged substrate $\left(f_{c}=1\right)$. The dependence of $I_{c}$ then follows from Eq. (1) by progressively moving the assumed front surface deeper into the damage layer.

The three different curves of Fig. 7 correspond to three choices for $f_{c}(0)$. All were normalized at a depth of 5000 $\AA$ to agree with the crystalline intensity. The simplest model (linear interpolation) for the transition region between the plateau and the pristine crystal was used; other depth dependences were considered, but the exact nature of Eq. (1) was not sensitive to the details of $f_{c}(x)$. As expressed in Eq. (4), $4200 \AA$ was used for the total damage depth and the plateau depth was $1600 \AA$.

In evaluating Eq. (1) for Fig. 7 we have neglected small changes in reflectance $(R)$ between damaged, etched, and crystalline samples, ${ }^{22}$ or changes in scattering volume due to variations in the refractive index $(n)$. This was justified by estimates of $R$ and $n$ obtained by means of the effective medium approximation. ${ }^{21}$ Dielectric function values were taken from Refs. 15 (crystal), 9, and 23 (amorphous). [Absorption coefficients generated in this manner are in good agreement with Eq. (3).] Changes in $R$ and $n$, over the range of $f_{c}$ considered, were found to yield negligible corrections.

The values of $f_{c}(0)$ used to generate the curves in Fig. 7 were $0.93,0.88$, and 0.75 for $5 \times 10^{13}, 1 \times 10^{14}$, and 
$5 \times 10^{14} \mathrm{~cm}^{-2}$, respectively. These values indicate that, even in the high-fluence implants, the sample remains predominantly crystalline although the average crystallites are quite small $(75 \%$ crystallinity in the plateau, with 60 - $\AA$ crystallites, at $5 \times 10^{14} \mathrm{~cm}^{-2}$ ).

Suppose we assume that each ion produces a cylindrical damage track of diameter $D$, in which amorphization occurs. Then, for fluence $F$, the statistical result for $f_{c}(0)$ is just $\exp (-F A)$, where $A=\pi D^{2} / 4$. (This is the uncovered fraction of a plane upon which overlappable disks of area $A$ are randomly placed with density $F$.) Using, for each $F$, the value of $f_{c}(0)$ obtained from the analysis of Fig. 7, this expression implies $D$ values of 4.3, 4.0, and $2.7 \AA$ for the three fluences. While these are only modestly consistent with each other, a result of $4 \AA$ for the damage track diameter is reasonable in view of the lightness of the $\mathrm{Be}^{+}$ion. For $\mathrm{As}^{+}$implanted into GaAs, Aspnes et al. ${ }^{24}$ estimated a track diameter upper limit of $10 \AA$.

Figure 8 displays our Raman-derived experimental results for the structural depth profile of the damage layer in implanted $\mathrm{GaAs}$, for the case of $45-\mathrm{keV} \mathrm{Be}^{+}$ $\left(5 \times 10^{14} \mathrm{~cm}^{-2}\right.$ results are shown). Data points symbolized by solid circles are reciprocal crystallite sizes obtained from the peak and linewidth results of Figs. 5 and 6. Those represented by an $\times$ are direct amorphous intensities (relative to $\mathrm{CaF}_{2}$ ) taken from full spectra $\left(20-300 \mathrm{~cm}^{-1}\right)$ and scaled to agree with the other results in Fig. 8. The heavy trapezoidal line is the amorphous fraction $1-f_{c}(x)$ which corresponds to the model used [Eq. (4)] to generate the fit to the intensity data of Fig. 7. The overall agreement of these three measures of implantation-induced disorder is very satisfactory and supports the mixed amorphous-microcrystalline model for the structure of the damage layer. These measures reveal a high-damage near-surface plateau which extends to a depth that is about a third of the full depth.

The picture which emerges of the near-surface damage layer is a region of constant average crystallite size and constant crystalline volume fraction coexisting with amorphous $\mathrm{GaAs}$ within a fine-scale two-phase matrix. This high-damage region extends approximately $1500 \AA$ from the surface for $45-\mathrm{keV}$ beryllium implants. A transition region follows in which $f_{c}$ and crystallite size grow steadily until the underlying single crystal is reached at approximately $4000 \AA$.

Figure 8 also includes, for comparison to the experimental results, two widely used theoretical estimates of damage profiles (shown for the case of $45-\mathrm{keV} \mathrm{Be}^{+}$into GaAs): (Lindhard-Scharff-Schiøtt theory) ${ }^{25}$ LSS and TRIM (Ref. 26) ("transfer of ions in matter," a Monte Carlo calculation of the displaced atoms). These are included as the curves in Fig. 8. The broad maximum in the TRIM calculation agrees fairly well with the extent of the high-damage plateau revealed by our experiments. The LSS maximum occurs near the center of the observed damage plateau. Both calculations underestimate the full depth of the damage. A dip in the damage density is predicted at the surface by both calculations, but it is not observed experimentally. This is not an artifact of the finite optical penetration depth. We also depth

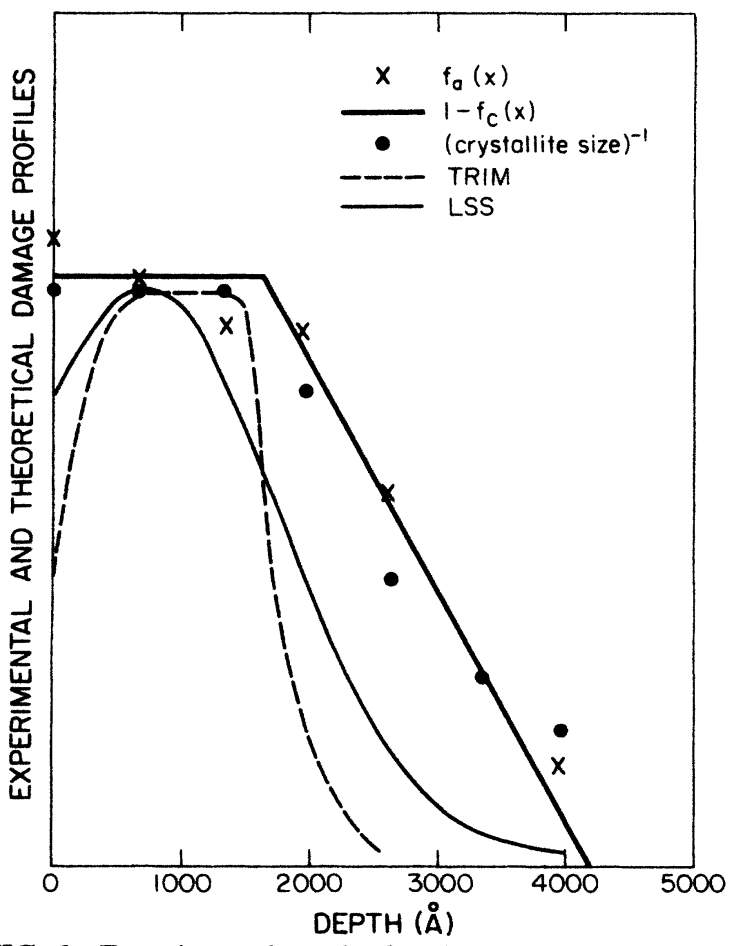

FIG. 8. Experimental results for the structural depth profile of ion-implanted GaAs $\left(45-\mathrm{keV} \mathrm{Be}{ }^{+}, 5 \times 10^{14}-\mathrm{cm}^{-2}\right.$ case). The heavy trapezoidal line labeled $1-f_{c}(x)$ is the amorphous volume fraction derived from the model used to fit the LO intensity data in Fig. 7. The crosses represent amorphous intensities, relative to $\mathrm{CaF}_{2}$, taken from amorphous bands as in Fig. 1. Solid circles denote reciprocal crystallite sizes obtained from the LO line-shape data of Figs. 5 and 6. The two curves are theoretical calculations included for comparison.

profiled with the $4579-\AA$ laser line, which has a penetration depth of only $260 \AA$ in crystalline GaAs (Ref. 15) and only $90 \AA$ in amorphous GaAs, ${ }^{9}$ both smaller than the etch step. These Raman experiments, which probed a layer substantially thinner than the etch step, yielded the same result: the high-damage plateau extends to the surface.

Christel and Gibbons ${ }^{27}$ applied the transport equation approach to $50-\mathrm{keV}$ boron in GaAs. They found a displacement density (As and $\mathrm{Ga}$ displacements) which increased toward the surface. Erman et al. ${ }^{10}$ depthprofiled, by chemical etching, high-fluence $10-\mathrm{keV} \mathrm{Be}^{+}$in GaAs using spectral ellipsometry. They observed a narrow damage plateau region at the surface which was about $40 \%$ amorphous, followed by a monotonic transition region to the crystal. Theeten and Erman' ${ }^{9}$ used spectral ellipsometry and the effective medium approximation to profile the damage induced by $10-\mathrm{keV} \mathrm{B}^{+}$in GaAs. Their results indicated a high damage density near the surface, although their profile could be interpreted ambiguously. The evidence for a near-surface damage plateau is not restricted to low-energy implants. Kwun et al. ${ }^{11}$ observed a similar damage profile, via the refractive index, for $250-\mathrm{keV}$ beryllium implants. The presence of a damage plateau (to $9000 \AA$ in their case) is not expected by LSS. Also, in our own laboratory, we have observed uniform damage up to at least $4000 \AA$ for $180-\mathrm{keV}$ 
$\mathrm{Be}^{+}$implants.

All of this supports the experimental evidence of Figs. 4-8 for a near-surface high-damage plateau region, and it contradicts the predictions of the LSS and TRIM simulations that there is a near-surface decrease in the degree of structural damage. The explanation for the inadequacy of the theoretical simulations, with respect to this point, is not understood and needs to be examined. It is in contrast to the success which these models have in predicting the concentration profile of the implanted dopant atoms. ${ }^{3}$ It appears that, for the host lattice, the concentration of displaced atoms is not a satisfactory measure of the actual disordered structure that is produced by implantation.

\section{THE EFFECT OF PHOTON ENERGY}

Optical penetration depth depends upon photon energy for an opaque semiconductor. Thus the depth probed by Raman scattering may be varied by changing the laser photon energy, $\hbar \omega_{L}$. This provides the possibility of a nondestructive probe of structural variations. In a simple two-layer material composed of structurally homogeneous layers, a simple superposition of spectra would be seen with the relative intensities of the separate spectral components varying with the depth probed. In our case we have a continuously graded material, with the additional complication of a spectral component, the crystallite-size-dependent LO line, which has a spectral shape that varies with depth. This makes the deconvolution of contributions from different depths more difficult. Fortunately, the broad-band continuum, which is the signature of amorphous GaAs, was found to be insensitive

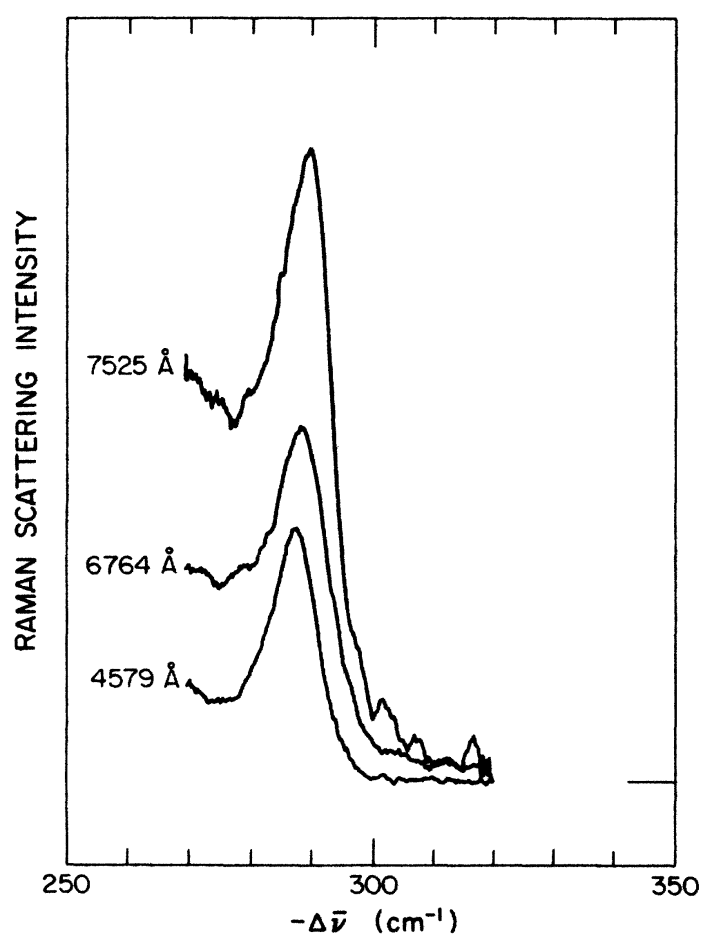

FIG. 9. The LO spectrum of the $5 \times 10^{14}-\mathrm{cm}^{-2}$ implant for a wide range of laser photon energies. All are plotted on the same scale, normalized to the $\mathrm{CaF}_{2}$ standard. to $\hbar \omega_{L}$, so that this spectral component could be treated as invariant in shape.

In Fig. 9 the spectrum in the neighborhood of the LO line, for the $5 \times 10^{14}-\mathrm{cm}^{-2}$ specimen (no etch), is plotted for laser photon energies of $1.65,1.83$, and $2.71 \mathrm{eV}$. All intensities are on the same absolute scale $\left(\mathrm{CaF}_{2}\right.$ standard), with the zero of intensity at $320 \mathrm{~cm}^{-1}$. These uncorrected spectra are slightly different in shape (when plotted with the same peak height). However, after subtracting the amorphous $\mathrm{GaAs}$ contribution to the spectrum, all three lineshapes are identical, which means that the same distribution of crystallite sizes is being probed. Thus the range of the probe does not extend beyond the highdamage plateau for these photon energies. The differences in intensity are then a matter of penetration depth and, as discussed below, resonance effects.

For the laser lines with $\hbar \omega_{L} \geq 1.84 \mathrm{eV}\left(\lambda_{L} \leq 6764 \AA\right)$, the LO line of the $5 \times 10^{14}-\mathrm{cm}^{-2}$ implant does not change as etch time changes from 0 to 1 to $2 \mathrm{~min}$. Thus, for these wavelengths, the penetration depth $d_{\text {opt }}$ is less than the depth corresponding to $1 \mathrm{~min}$ of etch $(660 \AA)$. However, for 1.65-eV (7525- $\AA$ ) excitation, the LO line shape observed after $1 \mathrm{~min}$ of etch time is appreciably different from that observed for the unetched sample. It follows that $d_{\text {opt }}$ at this $\hbar \omega_{L}$ matches the thickness removed after an etch time of between 2 and $3 \mathrm{~min}$. Using this procedure, along with the results of the previous section, and etch-profiling results obtained with other laser lines, it is possible to estimate $d_{\text {opt }}$, and thereby $\alpha(\hbar \omega)=1 /\left(2 d_{\text {opt }}\right)$, within the damage plateau region. Estimates of $\alpha$ for the $5 \times 10^{14}-\mathrm{cm}^{-2}$ specimen obtained by this line-shape analysis are $30 \pm 5 \times 10^{3} \mathrm{~cm}^{-1}(1.65 \mathrm{eV})$ and $80 \pm 10 \times 10^{3}$ $\mathrm{cm}^{-1}\left(1.9 \mathrm{eV}\right.$ ). Using Eq. (2) with $f_{c}=0.75$ (obtained from LO intensity measurements) we get $\alpha=37 \times 10^{3}$ $\mathrm{cm}^{-1}(1.65 \mathrm{eV})$ and $70 \times 10^{3} \mathrm{~cm}^{-1}(1.9 \mathrm{eV})$. These estimates are in reasonable agreement with each other. A

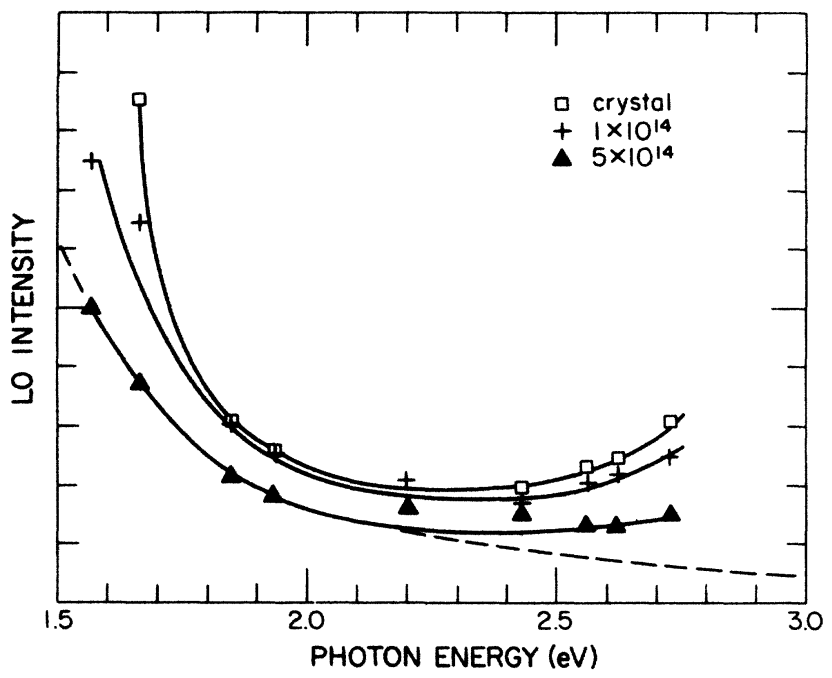

FIG. 10. LO integrated Raman intensities for crystalline GaAs and for the implants of 1 and $5 \times 10^{14} \mathrm{~cm}^{-2}$, over a wide range of photon energies. All are normalized to the $\mathrm{CaF}_{2}$ standard. The dashed curve shows the spectral dependence of the optical penetration depth for the high-implant sample. 
plot of $d_{\text {opt }}$ using Eq. (2) with $75 \%$ crystalline volume fraction is shown in Fig. 10 as a dashed curve. It is scaled using the $1.65-\mathrm{eV}$ LO intensity value for the $5 \times 10^{14}-\mathrm{cm}^{-2}$ implant.

Figure 10 shows the LO integrated intensity plotted against photon energy (i.e., the excitation spectrum for LO Raman emission) for the pristine crystal and for the implants of 1 and $5 \times 10^{14} \mathrm{~cm}^{-2}$. The left side of Fig. 10 reveals a strong increase in observed Raman signal at low $\hbar \omega$. Our etch-profile estimates for $d_{\text {opt }}(\hbar \omega)$ reveal that this increase in the LO intensity is well accounted for by the increase in penetration depth (dashed curve) with decreasing photon energy. However, Fig. 10 also reveals an increase in intensity at high photon energy. Careful measurements show that this is a real effect, strongest for the crystal and weaker for implanted material. For the crystal, the LO intensity increases by a factor of 1.3 between 2.5 and $2.7 \mathrm{eV}$, even though the optical penetration depth decreases by a factor of 1.7 over this range. Thus, although the scattering volume is decreasing with increasing $\hbar \omega$, the observed scattering intensity is increasing. We interpret this increase in scattering efficiency as a resonance-Raman effect.

For GaAs, a very strong resonance in the Raman scattering by TO phonons is observed, at room temperature, at $2.9 \mathrm{eV} .^{28}$ This is the energy of the strong $E_{1}$ electronic interband transition absorption peak in GaAs. (The $E_{0}$ interband transition at $1.43 \mathrm{eV}$, the optical band gap in GaAs, has much smaller oscillator strength and produces only very weak resonance. ${ }^{28}$ ) We attribute the increasing LO Raman-scattering efficiency with increasing photon energy, as seen in Fig. 10 for $\hbar \omega$ above $2.5 \mathrm{eV}$, to resonance with the approaching $E_{1}$ interband transition.

Figure 10 shows that the resonance-Raman effect at high $\hbar \omega$ is substantially diminished by implantation. We believe that this damage-induced quenching of the resonance enhancement is a consequence of the decreasing crystallite size. Decreasing crystallite size broadens the interband peaks in the electronic spectrum, ${ }^{24}$ and it is likely that this broadening of the initially sharp crystal peaks is the dominant effect in reducing the resonance. Reflectivity changes are not a factor; at $2.7 \mathrm{eV}$, the reflectance change caused by implantation is at most a few percent. ${ }^{10,29}$ Optical penetration depth is decreasing with $\hbar \omega$ throughout this region for both the crystal and the implanted material, more slowly for the latter. ${ }^{24}$ Since $I \sim d_{\mathrm{opt}}$, correcting for $d_{\mathrm{opt}}(\hbar \omega)$ increases both the enhancement effect for the scattering efficiency and the enhancement difference between the crystal and the implanted GaAs.

\section{SUMMARY}

A quantitative depth profile of the structural changes caused by high-fluence $45-\mathrm{keV}$ beryllium implants into GaAs has been obtained using Raman scattering in combination with chemical etch. The experimental observations are well accounted for by a structural model consisting of a fine-scale amorphous-microcrystal mix. Our results for the depth dependence of the amorphous volume fraction and the characteristic crystallite size have been presented in Fig. 8. Both measures present the same depth profile. A high-damage plateau region, in which these measures change little with depth, is followed by a transition region in which they vary smoothly until the bulk-crystal values are reached. There is no sign of a near-surface decrease in disorder, an experimental result which appears to disagree with LSS and TRIM theoretical predictions. Increasing the ion fluence, at constant ion energy, increases the amplitude of the damage (e.g., at $5 \times 10^{14} \mathrm{~cm}^{-2}$, the highest ion fluence studied, the amorphous fraction in the plateau region is 0.25 and the crystallite size is about $60 \AA$ ) but does not appreciably change the shape of the depth profile (Figs. 5, 6, and 7). An amorphization track diameter of about $4 \AA$ is estimated for $45-\mathrm{keV} \mathrm{Be}^{+}$ions incident upon GaAs.

The LO Raman line of the crystalline fraction was studied as a function of excitation photon energy from 1.55 to $2.71 \mathrm{eV}$. The LO intensity rises rapidly at low photon energies because of the increasing optical penetration depth. We also find, as seen in Fig. 10, that there is a real increase in the Raman-scattering efficiency above 2.5 $\mathrm{eV}$, an effect we attribute to resonance with the $E_{1}$ interband transition at $2.9 \mathrm{eV}$. This resonance-Raman effect is quenched by implantation, most probably because of the broadening of the $E_{1}$ crystal peak as the crystallite size decreases with increasing ion bombardment.

\section{ACKNOWLEDGMENTS}

The authors gratefully acknowledge the expert help of $T$. Brandon and $K$. Rice, and we have benefited from valuable discussions with L. C. Burton, J. G. Dillard, W. M. Duncan, G. F. Feng, and A. Purdes. This work was supported in part by Texas Instruments and the Virginia Center for Innovative Technology.
*Present address: Max-Planck-Institut für Festkörperforschung, Stuttgart.

$\dagger$ Permanent address: Physics Department and Solid State Institute, Technion, Haifa.

$\ddagger$ Present address: Department of Physics, University of North Texas, Denton, Texas 76203.

1J. Lindhard, M. Scharff, and H. E. Schiøtt, K. Dan. Vidensk. Selsk., Mat. - Fys. Medd. 33, 14 (1963).

2J. F. Ziegler, J. P. Biersack, and U. Littmark, The Stopping and Range of Ions in Solids (Pergamon, London, 1985).
${ }^{3}$ W. M. Duncan and G. Westphal in VLSI Electronics: Microstructure Science, edited by N. G. Einspruch and W. R. Wisseman (Academic, New York, 1985), p. 41.

${ }^{4}$ K. K. Tiong, P. M. Amirtharaj, F. H. Pollak, and D. E. Aspnes, Appl. Phys. Lett. 44, 122 (1984).

${ }^{5}$ T. Nakamura and T. Katoda, J. Appl. Phys. 53, 5870 (1982).

${ }^{6}$ C. S. Rama Rao, S. Sundaram, R. L. Schmidt, and J. Comas, J. Appl. Phys. 54, 1808 (1983).

${ }^{7}$ M. Holtz, R. Zallen, A. Geissberger, and R. Sadler, J. Appl. Phys. 59, 1946 (1986). 
${ }^{8}$ H. Richter, Z. P. Wang, and L. Ley, Solid State Commun. 39, 625 (1981).

${ }^{9}$ J. B. Theeten and M. Erman, J. Vac. Sci. Technol. 20, 471 (1982).

${ }^{10}$ M. Erman, J. B. Theeten, P. Chambon, S. M. Kelso, and D. E. Aspnes, J. Appl. Phys. 56, 2664 (1984).

${ }^{11}$ Sook-Il Kwun, M. H. Lee, L. L. Liou, W. G. Spitzer, H. L. Dunlap, and K. Vaidyanathan, J. Appl. Phys. 57, 1022 (1985).

${ }^{12}$ W. McLevige, Ph.D dissertation, University of Illinois, 1978, p. 17.

${ }^{13}$ S. Tolansky, Multiple-beam Interferometry of Surfaces and Films (Oxford University Press, London, 1947), p. 147.

${ }^{14} \mathrm{H}$. Ryssel and I. Ruge, Ion Implantation (Wiley, Chichester, 1986), p. 193.

${ }^{15}$ D. E. Aspnes and A. A. Studna, Phys. Rev. B 27, 985 (1983).

${ }^{16} \mathrm{~W}$. Hayes and R. Loudon, Scattering of Light by Crystals (Wiley, New York, 1978).

${ }^{17}$ M. Cardona, in Light Scattering in Solids, Vol. 2 of Topics in Applied Physics, edited by M. Cardona and G. Güntherodt (Springer-Verlag, Berlin, 1982), p. 45.

${ }^{18}$ R. Zallen, M. Holtz, R. A. Sadler, and A. E. Geissberger, Bull. Am. Phys. Soc. 31, 476 (1986).
${ }^{19}$ L. L. Abels, S. Sundaram, R. L. Schmidt, and J. Comas, Appl. Surf. Sci. 9, 2 (1981).

${ }^{20}$ Flash-evaporated amorphous GaAs films were provided by M-L. Theye, Université Pierre et Marie Curie, Paris; M-L. Theye and A. Gheorghiu, Sol. Energy Mater. 8, 331 (1982).

${ }^{21}$ D. E. Aspnes (unpublished).

${ }^{22}$ The etching process results in a slightly higher surface reflectance due possibly to oxide formation, or, more likely, to scratches and pitting. The damaged samples have a slightly higher reflectance $(2-4 \%)$.

${ }^{23}$ D. E. Aspnes (private communication).

${ }^{24}$ D. E. Aspnes, S. M. Kelso, C. G. Olson, and D. W. Lynch, Phys. Rev. Lett. 48, 1863 (1982).

${ }^{25} \mathrm{G}$. Carter and W. A. Grant, Ion Implantation of Semiconductors (Edward Arnold, London, 1976), p. 135.

${ }^{26}$ J. F. Ziegler, J. P. Biersack, and V. Littmark, The Stopping and Range of Ions in Solids, Ref. 2, p. 109.

${ }^{27}$ L. A. Christel and J. F. Gibbons, J. Appl. Phys. 52, 5050 (1981).

${ }^{28}$ M. H. Grimsditch, D. Olego, and M. Cardona, Phys. Rev. B 20, 1758 (1979).

${ }^{29}$ G. F. Feng (unpublished). 